\title{
Designing electron spin textures and spin interferometers by shape deformations
}

\author{
Zu-Jian Ying, ${ }^{1,2}$ Paola Gentile, ${ }^{1}$ Carmine Ortix,${ }^{3,4}$ and Mario Cuoco ${ }^{1}$ \\ ${ }^{1}$ CNR-SPIN and Dipartimento di Fisica “E. R. Caianiello," Università di Salerno, I-84084 Fisciano (Salerno), Italy \\ ${ }^{2}$ Beijing Computational Science Research Center, Beijing 100084, China \\ ${ }^{3}$ Institute for Theoretical Solid State Physics, IFW-Dresden, Helmholtzstraße 20, D-01069 Dresden, Germany \\ ${ }^{4}$ Institute for Theoretical Physics, Center for Extreme Matter and Emergent Phenomena, Utrecht University, \\ Princetonplein 5, 3584 CC Utrecht, The Netherlands
}

(Received 7 March 2016; revised manuscript received 26 May 2016; published 11 August 2016)

\begin{abstract}
We demonstrate that the spin orientation of an electron propagating in a one-dimensional nanostructure with Rashba spin-orbit (SO) coupling can be manipulated on demand by changing the geometry of the nanosystem. Shape deformations that result in a nonuniform curvature give rise to complex three-dimensional spin textures in space. We employ the paradigmatic example of an elliptically deformed quantum ring to unveil the way to get an all-geometrical and all-electrical control of the spin orientation. The resulting spin textures exhibit a tunable topological character with windings around the radial and the out-of-plane directions. We show that these topologically nontrivial spin patterns affect the spin interference effect in the deformed ring, thereby resulting in different geometry-driven ballistic electronic transport behaviors. Our results establish a deep connection between electronic spin textures, spin transport, and the nanoscale shape of the system.
\end{abstract}

DOI: 10.1103/PhysRevB.94.081406

Introduction. The manipulation and control of the electron spin are essential ingredients for the development of innovative quantum-engineered devices [1-3]. The spin-orbit (SO) coupling [4-6] is particularly attractive in this framework because it offers the promising prospect [7] of an all-electrical intrinsic control over the spin without applying a magnetic field. For low-dimensional nanosystems with structure inversion asymmetry, this coupling between the orbital motion and the electron spin is due to the so-called Rashba SO interaction [4-6]. The Rashba SO coupling is at the heart of a growing research interest for the spin generation, manipulation, and detection due to the tantalizing possibilities of tuning the spin orientation through the electron propagation and vice versa to exert a spin control of the electron trajectories. Central consequences of these constituent features are, among the many possibilities, the spin Hall $[8,9]$ and the spin galvanic effects [10-12], the SO driven spin torque [13,14], the design of quantum topological states [15], etc.

Charge carriers in materials with Rashba SO are subject to a momentum dependent effective magnetic field which not only results in spin-dependent velocity but also in a nontrivial geometric phase. Such phase is another important means to manipulate the electron spin. For instance, in the Aharonov-Casher (AC) effect [16], magnetic dipoles moving around a tube of electric charge acquire a nontrivial Berry [17] or geometric phase [18], being similar to the case of the Aharonov-Bohm [19] effect for charged particles moving in a closed circuit around a magnetic flux. With the development of semiconducting nanostructures, a possibility has emerged to tune quantum states by combining Rashba effects and geometric phases. Considering the role of magnetic field

Published by the American Physical Society under the terms of the Creative Commons Attribution 3.0 License. Further distribution of this work must maintain attribution to the author(s) and the published article's title, journal citation, and DOI. textures in driving a spin geometric phase [20], the effects of quantum geometric phases have been predicted [21] and experimentally observed in the transport properties of semiconducting quantum rings [22,23]. Such findings opened the path to a spin topological design [24], as demonstrated by the magnetic field driven topological transition in the geometric phase and the consequences on the spin transport $[23,25]$.

In view of the rapid progress in nanostructuring techniques, it would be highly challenging and desirable to design nanostructures where the system geometry is the main knob to tailor the electron spin properties. In this Rapid Communication, we demonstrate that the curvature of a nanostructure with Rashba SO coupling allows one to manipulate and control the spin orientation and the spin transport. We use the prototypical geometry of elliptical quantum rings to unveil complex three-dimensional spin textures in space and how they evolve in the presence of a nonuniform curvature. The resulting spin patterns have distinct topological features with tunable windings around the radial and the out-of-plane spin directions along the elliptical ring. Remarkably, we find different types of transitions between quantum states with inequivalent spin textures by varying the curvature strength or the Rashba SO coupling thus indicating a unique path to control the electron spin in curved nanostructures. We then demonstrate a fundamental twist between curvature, spin texture, and spin transport

Curvature-driven spin torques. Electrons confined to move along a one-dimensional planarly curved nanostructure [Fig. 1(a)] are subject to a Rashba SO interaction, due to the inversion symmetry breaking that, as for the case of a straight nanostructure, couples the orbital momentum and the local spin component that is normal to the electron motion [26]. Since the nanostructure has a nontrivial curvature, the local normal spin direction depends on the position and thus generally it does not commute with the momentum. Such interplay can be conveniently expressed by introducing the local normal $\hat{\mathcal{N}}(s)$ and tangential $\hat{\mathcal{T}}(s)$ directions at a given position $s$ along the curve, as well as the related local Pauli matrices $\sigma_{N}(s)=\tau \cdot \hat{\mathcal{N}}(s)$ and $\sigma_{T}(s)=\tau \cdot \hat{\mathcal{T}}(s)$ 

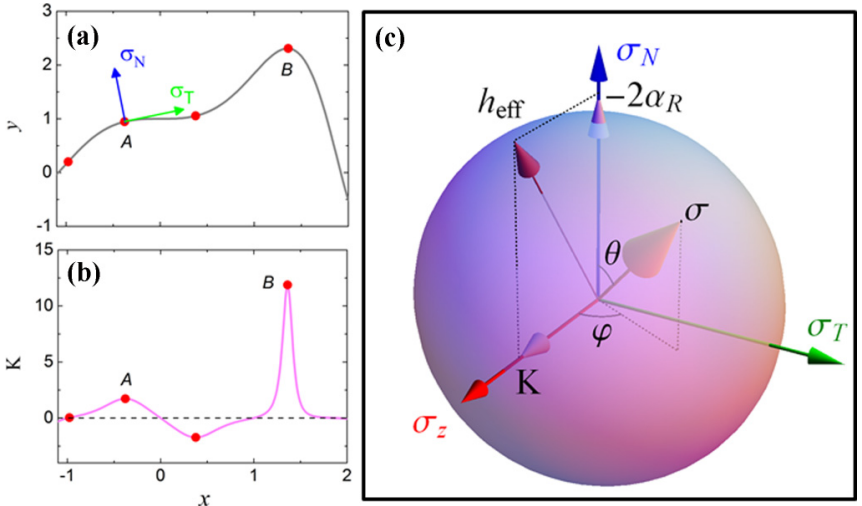

FIG. 1. Schematics of (a) the profile of a planarly curved nanowire and (b) the related curvature $K(s)$. (c) denotes the FrenetSerret-Bloch sphere in the moving frame of the electrons with the vectors associated to the electron spin orientation and the effective field $\boldsymbol{h}_{\text {eff }}$.

in the moving frame of the electrons, with $\tau$ being the usual Pauli matrices [Fig. 1(a)]. Then, the Hamiltonian for a planar nonuniformly curved nanostructure with Rashba SO can be written as [26-29]

$$
\mathcal{H}_{\mathbf{k} \cdot \mathbf{p}}=-\frac{\hbar^{2}}{2 m^{\star}} \partial_{s}^{2}+\frac{i \alpha_{S O}}{2}\left[\sigma_{N}(s) \partial_{s}+\partial_{s} \sigma_{N}(s)\right],
$$

where $s$ is the arclength of the planar curve measured from an arbitrary reference point, $m^{\star}$ is the effective mass of the charge carriers, and $\alpha_{S O}$ is the Rashba SO coupling strength. Equation (1) generalizes the Hamiltonian originally proposed for a quantum ring with constant curvature [30]. It is convenient to express the normal and tangential directions to the curve in terms of a polar angle $f(s)$ as $\hat{\mathcal{N}}(s)=\{\cos f(s)$, sin $f(s), 0\}$, and $\hat{\mathcal{T}}(s)=\{\sin f(s),-\cos f(s), 0\}$. Using the Frenet-Serret (FS) type equation of motion [31], $\partial_{s} \hat{\mathcal{N}}(s)=K(s) \hat{\mathcal{T}}(s)$, where $K(s)$ is the local curvature, we can immediately relate the polar angle to the local curvature via $\partial_{s} f(s)=-K(s)$ [Figs. 1(a) and 1(b)]. To proceed further, we use the fact that a spin eigenmode $\left|\Psi_{E}\right\rangle$ of the Hamiltonian in Eq. (1) evolves in space according to $i \partial_{s}\left|\Psi_{E}\right\rangle=G(s)\left|\Psi_{E}\right\rangle$ where $G(s)=$ $-\left[\alpha_{R} \sigma_{N}(s)+c_{0} \sigma_{0}\right], \alpha_{R}=\frac{m^{*} \alpha_{S O}}{\hbar^{2}}$ being the inverse Rashba SO length, while $c_{0}^{2}=\frac{2 m^{*} E}{\hbar^{2}}+\alpha_{R}^{2}$ depends upon the eigenergy $E$. This relation allows us to derive the spin torque exerted on the electron spin through a fundamental equation that links the geometric curvature of the nanostructure, the Rashba SO coupling, and the electron spin orientation in the FS frame [32]:

$$
\partial_{s}\langle\boldsymbol{\sigma}\rangle=\boldsymbol{h}_{\text {eff }} \times\langle\boldsymbol{\sigma}\rangle .
$$

Here, the local spin orientation is $\langle\boldsymbol{\sigma}\rangle=\left\{\left\langle\sigma_{T}\right\rangle,\left\langle\sigma_{N}\right\rangle,\left\langle\sigma_{z}\right\rangle\right\}$ while the effective spin-orbit field $\boldsymbol{h}_{\text {eff }}=\left\{0,-2 \alpha_{R}, K(s)\right\}$ lies in the normal-binormal plane and depends on the local curvature and $\alpha_{R}$. The spin direction then defines a FrenetSerret-Bloch (FSB) sphere which is expressed in terms of the azimuthal and polar angles $\theta(s)$ and $\varphi(s)$ [Fig. 1(c)]. Equation (2) generally implies that due to a nonzero curvature, the electron spin acquires a finite out-of-plane binormal $\hat{z}$ component. More importantly, a nontrivial component along the tangential direction appears provided the curvature is not

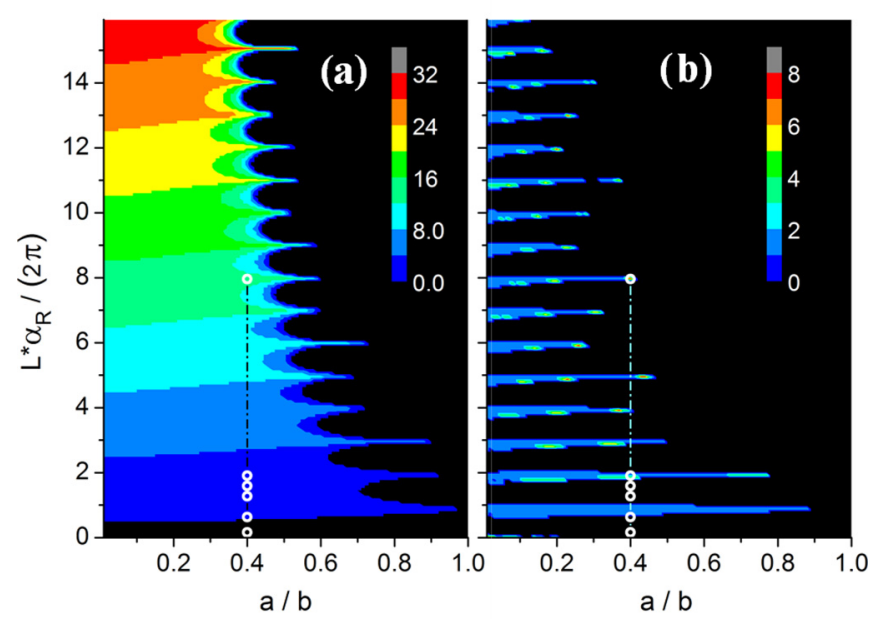

FIG. 2. Contour map of the winding number of the local spin orientation around (a) the normal $N$ direction in the moving frame and (b) the out-of-plane $z$ axis as a function of the ellipse ratio $a / b$ and the scaled inverse SO length $L \alpha_{R} /(2 \pi)$. The white circles at $a / b=0.4$ indicate specific points of presented spin textures (see Fig. 3).

constant. Albeit the derivative $\partial_{s}$ of the spin vector locally vanishes if the spin is aligned to the effective spin-orbit field, the variation of the local curvature yields a nonvanishing torque which results in a component of the spin vector parallel to the electron propagation direction. Insights into the electron spin trajectories regulated by Eq. (2) can be then gained by analyzing the spin velocity vector field around the $\boldsymbol{h}_{\text {eff }}$ direction on the FSB sphere [32].

Elliptical quantum rings. We next investigate the spin texture in a nanostructure with nonuniform curvature by considering the example of a quantum ring of length $L$ with an elliptical shape and a ratio $a / b$ between the minor $(a)$ and the major $(b)$ axes of the ellipse. This is a paradigmatic case of a nanostructure with positive but nonuniform curvature that can be suitably enhanced (suppressed) at the positions nearby the poles of the major (minor) axes. In order to single out the spin textures of the eigenmodes, we introduce a tight-binding model obtained by discretizing Eq. (1) on a lattice [32]. We then characterize "topologically" the resulting spin texture by counting the number of windings around the normal $\hat{N}$ and the binormal $\hat{z}$ directions the spin traces over the FSB sphere in a single loop. As shown in Fig. 2, we observe two distinct spin texture regimes. There is a region of the parameter space (black area), corresponding to configurations with strong SO or quasiconstant curvature, where the electron spin is pinned nearby the quasistatic effective field $\boldsymbol{h}_{\text {eff }}$ and thus does not exhibit any winding in the FSB sphere. For $a / b$ below $\sim 0.5$, i.e., for a sizable nonuniform curvature profile, the electron spin is not able to follow the periodic motion of the effective spin-orbit field and manifests windings both around the normal and the binormal directions. It follows that one can switch from a configuration without any winding to another with winding around the normal $\hat{N}$ and/or the binormal $\hat{z}$ directions through a pure geometric effect by tuning the ratio $a / b$ or by an all-electrical control of the Rahsba SOC. 


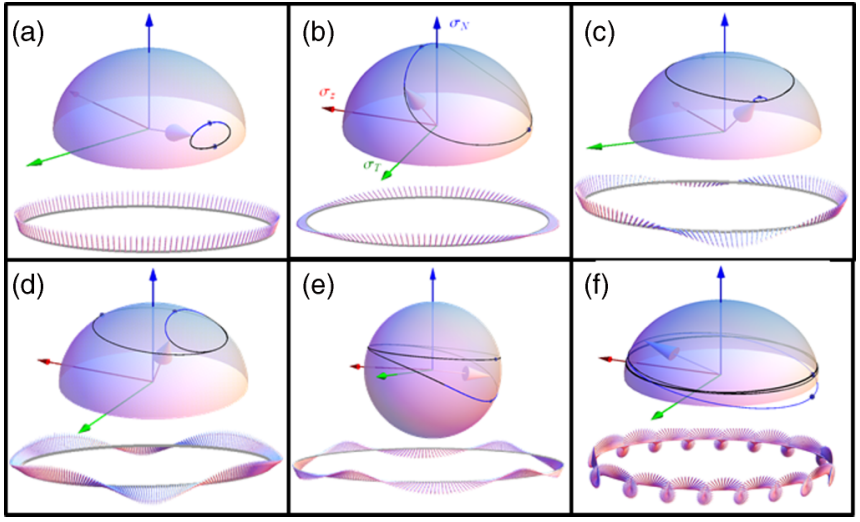

FIG. 3. Evolution of the spin orientation trajectories on the Bloch sphere and of the three-dimensional spin textures along the ellipse shaped ring at a given ratio $a / b=0.4$ and for various values of the SO coupling (white circles in Fig. 2), i.e., $L \alpha_{R}=1,4,8,10,12,50.1$ corresponding to the panels (a)-(f), respectively. The blue portion of the trajectory stands for the part of the elliptical loop with larger curvature. The dots mark the positions on the loop with the maximum and minimum values of the local curvature.

Remarkably, the onset of the spin textures with a finite number of windings can be achieved not only via a shape deformation, but also by an all-electrical control of the Rahsba spin-orbit inverse length $\alpha_{R}$. Below a critical threshold of the $a / b$ ratio, indeed, we find that an increase in $\alpha_{R}$ has a twofold effect. First, it leads to an increase in the winding number around the normal direction that clearly exhibits constant plateaus [Fig. 2(a)]. Second, the winding number around the binormal direction has an irregular comblike structure with a switchable on and off behavior that is driven by a small variation of the Rashba spin-orbit inverse length [Fig. 2(b)]. To highlight the features of the spin textures we also explicitly show in Fig. 3 the spin trajectories in the FSB frame and the spin textures in the loop reference frame for few representative values of the $a / b$ and $\alpha_{R}$ parameters.

Geometric control of the spin transport. The geometrical and the dynamical quantum phases are the key physical quantities that determine spin interference effects and thus the electronic transport in a cyclic quantum motion. From the spin-torque equation [Eq. (2)], we can derive the nonadiabatic Aharonov-Anandan (AA) [18] geometric phase $g_{A A}$, as well as the dynamical phase $d$ acquired by an electron moving along a closed loop as [32]

$$
\begin{aligned}
g_{A A} & =\pi\left(1+\frac{1}{2 \pi} \int q_{N T} d s-\frac{1}{2 \pi} \int \frac{\left\langle\sigma_{z}\right\rangle}{\rho}\left[K(s)+q_{N T}\right] d s\right), \\
d & =d_{\sigma}+d_{E},
\end{aligned}
$$

where $\rho$ is the local density of the spin eigenmode, $q_{N T}=\left[\left\langle\sigma_{N}\right\rangle \partial_{s}\left\langle\sigma_{T}\right\rangle-\left\langle\sigma_{T}\right\rangle \partial_{s}\left\langle\sigma_{N}\right\rangle\right] /\left[\left\langle\sigma_{T}\right\rangle^{2}+\left\langle\sigma_{N}\right\rangle^{2}\right]$, while $d_{\sigma}=\int \alpha_{R} \frac{\left\langle\sigma_{N}\right\rangle}{\rho} d s$ and $d_{E}=c_{0} L$ are the spin and energy dependent components of the dynamical phase. From Eq. (3) it follows that the nature of the spin texture affects the geometric and the dynamical phase in a very different manner. The geometric AA phase is half the solid angle spanned by

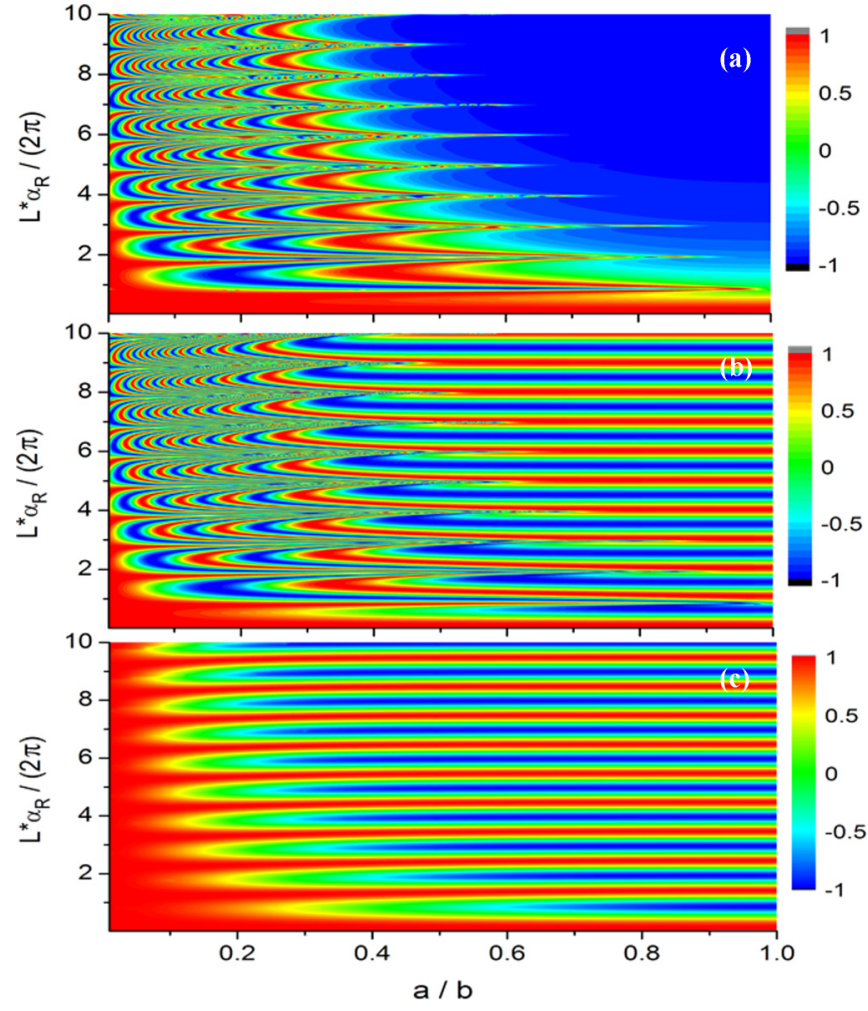

FIG. 4. Contour map of the cosine of the geometric phase (a), the spin component of the dynamical phase (b), and the total phase (c) contributing to the conductance as a function of the ellipse ratio $a / b$ and the scaled spin-orbit coupling $L \alpha_{R} /(2 \pi)$.

the spin vector and consists of three terms: the first $\pi$ term is the adiabatic spin geometric phase the electron would acquire when pinned at the pole of the FSB sphere, i.e., along the direction of the effective spin-orbit field. The second term corresponds, in units of $\pi$, to the winding number of the normal and tangential spin components around the out-of-plane direction. Due to the reflection symmetry of the ring with respect to its major axis, the latter is always an even integer, and therefore does not yield parity changes of the AA phase. Finally, the third term is the nonadiabatic part of the AA phase, being directly proportional to the tilt of the electron spin in the out-of-plane $\hat{z}$ direction [21,23]. For a circular quantum ring with $a / b \equiv 1$ this term is gradually suppressed as the Rashba SO inverse length increases, and thus the geometric phase reduces to the adiabatic $\pi$ spin geometric Berry phase. An analogous behavior occurs also in the case of elliptical quantum rings [the blue area in Fig. 4(a)] in the parameter space region where the spin orientation is pinned close to the quasistatic effective SO field direction. However, as soon as the electronic spin starts to wind, the contributions in the nonadiabatic term yield rapid variations of the spin geometric phase as either the $a / b$ ratio or $\alpha_{R}$ are changed. A closer inspection of the nonadiabatic terms in $g_{A A}$ reveals that the contribution proportional to $\sim\left\langle\sigma_{z}\right\rangle q_{N T}$ induces a cascade of phase slips, and therefore it represents the major player for the rapid variations of the spin geometric phase in Fig. 4(a). A similar behavior is also obtained for the spin dependent part of the dynamical phase 
that exhibits a typical oscillating behavior at a given $a / b$ as a function of the Rashba SO in the adiabatic regime for $a / b \sim 1$, while it turns into rapidly phase fluctuating patterns in the nonadiabatic region with winding of the spin textures [Fig. 4(b)].

The geometrical and dynamical phases yield the interference pattern in the quantum conductance. As for the case of the conventional ring [21,23,33], the conductance of a single ballistic elliptical ring symmetrically coupled to two contact leads can be obtained by means of the Landauer approach and it is given by $G=\frac{e^{2}}{h}\left[1+\cos \left(d_{\sigma}+g_{A A}\right)\right]$ [32]. Remarkably, we observe that in the nonadiabatic regime with topological nontrivial spin textures the interference between the geometrical and dynamical phases leads to a smooth behavior of the conductance. The resulting pattern is marked by distinct geometrically driven channels of electronic transport with an almost constant conductance or exhibiting a changeover from destructive to constructive interference as the $a / b$ ratio decreases [Fig. 4(c)]. From the analysis of the phase interference in the conductance we observe that the regular pattern of the nonadiabatic regime mainly arises from the out-of-plane spin component due to a cancellation between the winding term in $g_{A A}$ and the dynamical phase $d_{\sigma}$. It is then the spatial behavior of $\left\langle\sigma_{z}\right\rangle$ that dictates the conductance interference pattern by allowing a $\pi$ shift in the region of the parameter space where both a winding around the radial and the binormal directions occurs. Such windings make the spin spanning a solid angle with a large amplitude on the Frenet-Serret-Bloch sphere thus reducing the adiabatic $\pi$ contribution of the geometric phase. This result sets a tight connection between the changeover of the conductance and the topological character of the spin texture as one can directly observe by comparing the phase diagram in Fig. 2 and the patterns in Fig. 4(c).

Our predictions can be readily accessible in the laboratory taking advantage of the experimental advances in probing the time-reversal AC effect [22,34] in arrays of InAlAs/InGaAs quantum rings [22]. In such arrays, quantum rings have a typical length $L$ in the range of [3,6] $\mu \mathrm{m}$, and $\alpha_{S O} \sim 10^{-12} \mathrm{eV} \mathrm{m}$. Considering an effective electron mass $m^{*} \sim 0.07 m_{e}$, we have $L \alpha_{R} /(2 \pi) \in[0.5,1.0]$. By further taking into account the gate tunability of $\alpha_{S O}[22,35]$, it is possible to access regimes of $L \alpha_{R} /(2 \pi)$ in the range of $\sim[0.5,4]$, which allows one to explore the main features of the phase diagram.

One could then design an array of elliptically shaped rings, instead of circular ones, and use the ratio $a / b$ as an additional knob for the gate voltage dependence of the AC effect. A genuine consequence of the nonuniform curvature is the occurrence of a transient with a weakly amplitude-modulated conductance before the AC oscillatory behavior sets in. As a manifestation of the strength of the curvature gradient such regime exhibits a strong gate dependence via the Rashba SO coupling. In addition, and as can be seen from the horizontal stripes of Fig. 4(c), the AC oscillation frequency tends to the frequency of conventional circular rings in the large $\alpha_{R}$ limit. Therefore knowledge of the frequency in an ensemble of elliptical quantum rings can be used to extract the strength of the Rashba spin-orbit coupling with extreme precision.

Finally, by also considering that, apart from conventional material geometries, nanostructuring methods have recently achieved a level of control that even enables the synthesis of complex three-dimensional nanoarchitectures resembling biological structures [36-39], our findings anticipate an unbound potential for new device concepts of flexible spin orbitronics where the electron spin and the electronic transport are directly controlled by the system geometry.

Acknowledgments. We acknowledge the financial support of the Future and Emerging Technologies (FET) programme under FET-Open Grant No. 618083 (CNTQC). C.O. thanks the Deutsche Forschungsgemeinschaft (Grant No. OR 404/1-1) for support. We are thankful to D. Frustaglia for valuable discussions.
[1] D. D. Awschalom, L. C. Bassett, A. S. Dzurak, E. L. Hu, and J. R. Petta, Science 339, 1174 (2013).

[2] I. Žutic, J. Fabian, and S. Das Sarma, Rev. Mod. Phys. 76, 323 (2004).

[3] C. Chappert, A. Fert, and F. N. Van Dau, Nat. Mater. 6, 813 (2007).

[4] G. Dresselhaus, Phys. Rev. 100, 580 (1955).

[5] E. I. Rashba, Sov. Phys. Solid State 2, 1109 (1960).

[6] Y. A. Bychkov and E. I. Rasbha, Pis'ma Zh. Eksp. Teor. Fiz. 39, 66 (1984) [JETP Lett. 39, 78 (1984)].

[7] A. Manchon, H. C. Koo, J. Nitta, S. M. Frolov, and R. A. Duine, Nat. Mater. 14, 871 (2015).

[8] M. I. Dyakonov and V. I. Perel, Zh. Eksp. Teor. Fiz. Pis. Red. 13, 657 (1971) [JETP Lett. 13, 467 (1971)].

[9] J. E. Hirsch, Phys. Rev. Lett. 83, 1834 (1999).

[10] E. L. Ivchenko and G. E. Pikus, Pis'ma Zh. Eksp. Teor. Fiz. 27, 640 (1978) [JETP Lett. 27, 604 (1978)].

[11] S. D. Ganichev, Int. J. Mod. Phys. B 22, 1 (2008).

[12] V. M. Edelstein, Solid State Commun. 73, 233 (1990).

[13] B. A. Bernevig and O. Vafek, Phys. Rev. B 72, 033203 (2005).

[14] A. Manchon and S. Zhang, Phys. Rev. B 78, 212405 (2008).
[15] M. Z. Hasan and C. L. Kane, Rev. Mod. Phys. 82, 3045 (2010). [16] Y. Aharonov and A. Casher, Phys. Rev. Lett. 53, 319 (1984).

[17] M. V. Berry, Proc. R. Soc. London, Ser. A 392, 45 (1984).

[18] Y. Aharonov and J. Anandan, Phys. Rev. Lett. 58, 1593 (1987).

[19] Y. Aharonov and D. Bohm, Phys. Rev. 115, 485 (1959).

[20] D. Loss, P. Goldbart, and A. V. Balatsky, Phys. Rev. Lett. 65, 1655 (1990).

[21] D. Frustaglia and K. Richter, Phys. Rev. B 69, 235310 (2004).

[22] F. Nagasawa, J. Takagi, Y. Kunihashi, M. Kohda, and J. Nitta, Phys. Rev. Lett. 108, 086801 (2012).

[23] F. Nagasawa, D. Frustaglia, H. Saarikoski, K. Richter, and J. Nitta, Nat. Commun. 4, 2526 (2013).

[24] Y. Lyanda-Geller, Phys. Rev. Lett. 71, 657 (1993).

[25] H. Saarikoski, J. E. Vázquez-Lozano, J. P. Baltanás, F. Nagasawa, J. Nitta, and D. Frustaglia, Phys. Rev. B 91, 241406(R) (2015).

[26] P. Gentile, M. Cuoco, and C. Ortix, SPIN 03, 1340002 (2013).

[27] C. Ortix, Phys. Rev. B 91, 245412 (2015).

[28] P. Gentile, M. Cuoco, and C. Ortix, Phys. Rev. Lett. 115, 256801 (2015). 
[29] E. Zhang, S. Zhang, and Q. Wang, Phys. Rev. B 75, 085308 (2007).

[30] F. E. Meijer, A. F. Morpurgo, and T. M. Klapwijk, Phys. Rev. B 66, 033107 (2002).

[31] R. Dandoloff, R. Balakrishnan, and A. R. Bishop, J. Phys. A: Math. Gen. 25, L1105 (1992).

[32] See Supplemental Material at http://link.aps.org/supplemental/ 10.1103/PhysRevB.94.081406 for the derivation of the gyroscope-like equation for the spatial evolution of the local spin orientation in a generic planarly curved nanowire with Rashba spin-orbit coupling. We demonstrate how the spin velocity vector field behaves around the positions corresponding to the locally vanishing effective spin-orbit torque. Then, we derive the expression for the geometric phase acquired by an electron going around a loop having a nontrivial geometric curvature and Rashba spin-orbit coupling. We derive the expression for the quantum conductance of an elliptical ring symmetrically coupled to two contact leads. Finally, we provide the details for the continuum-to-lattice mapping of the Hamiltonian for a curved nanowire and the methodology applied to determine the phase diagrams.

[33] D. Bercioux, D. Frustaglia, and M. Governale, Phys. Rev. B 72, 113310 (2005).

[34] C. P. Umbach, C. Van Haesendonck, R. B. Laibowitz, S. Washburn, and R. A. Webb, Phys. Rev. Lett. 56, 386 (1986).

[35] D. Liang and X. P. A. Gao, Nano Lett. 12, 3263 (2012).

[36] V. Y. Prinz, V. A. Seleznev, A. K. Gutakovsky, A. V. Chehovskiy, V. V. Preobrazhenskii, M. A. Putyato, and T. A. Gavrilova, Physica E (Amsterdam) 6, 828 (2000).

[37] O. G. Schmidt and K. Eberl, Nature (London) 410, 168 (2001).

[38] H.-F. Zhang, C.-M. Wang, and L.-S. Wang, Nano Lett. 2, 941 (2002).

[39] S. Xu, Z. Yan, K.-I. Jang et al., Science 347, 154 (2015). 